\title{
VLBI observations of SN 2008iz
}

\section{Expansion velocity and limits on anisotropic expansion}

\author{
A. Brunthaler ${ }^{1}$, I. Martí-Vidal ${ }^{1}$, K. M. Menten ${ }^{1}$, M. J. Reid ${ }^{2}$, C. Henkel ${ }^{1}$, G. C. Bower ${ }^{3}$, H. Falcke ${ }^{4,5}$, H. Feng ${ }^{6}$, \\ P. Kaaret ${ }^{7}$, N. R. Butler ${ }^{3}$, A. N. Morgan ${ }^{3}$, and A. Weiß ${ }^{1}$ \\ 1 Max-Planck-Institut für Radioastronomie, Auf dem Hügel 69, 53121 Bonn, Germany \\ e-mail: brunthal@mpifr-bonn.mpg.de \\ 2 Harvard-Smithsonian Center for Astrophysics, 60 Garden Street, Cambridge, MA 02138, USA \\ 3 UC Berkeley, 601 Campbell Hall, Astronomy Department \& Radio Astronomy Lab, Berkeley, CA 94720, USA \\ 4 Department of Astrophysics, Radboud Universiteit Nijmegen, Postbus 9010, 6500 GL Nijmegen, The Netherlands \\ ASTRON, Postbus 2, 7990 AA Dwingeloo, The Netherlands \\ ${ }^{6}$ Department of Engineering Physics and Center for Astrophysics, Tsinghua University, Beijing 100084, PR China \\ 7 Department of Physics and Astronomy, University of Iowa, Van Allen Hall, Iowa City, IA 52242, USA
}

Received 26 January 2010 / Accepted 25 March 2010

ABSTRACT

\begin{abstract}
We present observations of the recently discovered supernova 2008iz in M 82 with the VLBI High Sensitivity Array at $22 \mathrm{GHz}$, the Very Large Array at frequencies of 1.4, 4.8, 8.4, 22 and $43 \mathrm{GHz}$, and the Chandra X-ray observatory. The supernova was clearly detected in two VLBI images separated by 11 months. The source shows a ring-like morphology and expands with a velocity of $\sim 23000 \mathrm{~km} \mathrm{~s}^{-1}$. The most likely explosion date is in mid-February 2008. The measured expansion speed is a factor of $\sim 2$ higher than expected under the assumption that synchrotron self-absorption dominates the light curve at the peak, indicating that this absorption mechanism may not be important for the radio emission. We find no evidence for an asymmetric explosion. The VLA spectrum shows a broken power law, indicating that the source was still optically thick at $1.4 \mathrm{GHz}$ in April 2009. Finally, we report upper limits on the X-ray emission from SN 2008iz and a second radio transient recently discovered by MERLIN observations.
\end{abstract}

Key words. stars: supernovae: general - stars: supernovae: individual: SN 2008iz - radio continuum: general galaxies: individual: M 82

\section{Introduction}

Radio supernovae (RSNe) are rare events and are difficult to study. So far, only about two dozen have been detected (e.g. Weiler et al. 2002) and most of them are distant and weak. Few radio supernovae have been imaged with Very Long Baseline Interferometry (VLBI) techniques, and the evolution of their expanding shells has been studied in only four sources (SN 1979C, SN 1986J, SN 1987A, SN 1993J). The best studied radio supernova so far is SN 1993J in M 81. Following the expansion of the supernova allowed many different phenomena to be studied (Marcaide et al. 1997, 2009a; Bietenholz et al. 2001, 2003; Pérez-Torres et al. 2001, 2002a; Bartel et al. 2002, 2007), including a measurement of the expansion speed, the deceleration of the shock front, and the proper motion of the supernova shell, for which a limit was obtained.

The recent discovery of a new bright radio supernova in M 82, SN 2008iz, (Brunthaler et al. 2009a,b) at a similar distance as SN 1993J offers the rare opportunity to study the evolution of another supernova in great detail and to make a comparison to SN 1993J. Prior to the results presented here, SN 2008iz has only been detected in the radio band with the VLA at $22 \mathrm{GHz}$ (Brunthaler et al. 2009a), MERLIN at $5 \mathrm{GHz}$ (Beswick et al. 2009), and the Urumqi telescope at $5 \mathrm{GHz}$ (Marchili et al. 2010). There have been no reported detections in visible light and Fraser et al. (2009) report only a non detection in the near infrared on 2009 June 11 . The non-detections at other wavebands indicate that the supernova exploded behind a large gas or dust cloud in the central part of M 82. Thus, it has not been possible to classify this supernova. However, since type Ia supernovae are not known to show strong radio emission, SN 2008iz is most likely a core collapse supernova, i.e. type Ib/c or II.

Here we present the first VLBI images of SN 2008iz taken $\sim 2.5$ and $\sim 13.5$ month after the explosion, a radio spectrum (at an age of $\sim 14.5$ month) from 1.4 to $43 \mathrm{GHz}$ and Chandra $\mathrm{X}$-ray observations. Throughout the paper, we adopt a distance of 3.6 Mpc (based on a Cepheid distance to M 81 determined by Freedman et al. 1994).

\section{Observations and data reduction}

\subsection{High sensitivity array observations at $22 \mathrm{GHz}$}

M 82 was observed using the High Sensitivity Array including the $\mathrm{NRAO}^{1}$ Very Long Baseline Array (VLBA), the Very Large Array (VLA), the Green Bank Telescope (GBT), and the Effelsberg $100 \mathrm{~m}$ telescope under project code BB255 on 2008 May 03 and 2009 April 08. The total observing time at each

1 The National Radio Astronomy Observatory is a facility of the National Science Foundation operated under cooperative agreement by Associated Universities, Inc. 
epoch was $12 \mathrm{~h}$. We used M $81^{*}$, the nuclear radio source in M 81, as the phase calibrator and switched between M $81^{*}$, $\mathrm{M} 82$, and 3 extragalactic background quasars every $50 \mathrm{~s}$ in the sequence M 81* - 0945+6924-M 81* - 0948+6848-M 81*M $82-1004+6936-\mathrm{M} \mathrm{81*}$, yielding a total integration time of $\sim 100 \mathrm{~min}$ on M 82. J1048+7143 was observed every $\sim 30 \mathrm{~min}$ to phase-up the VLA, and DA193 was observed as fringe finder. The data were recorded with four $8 \mathrm{MHz}$ frequency bands in dual circular polarization, with Nyquist sampling and 2 bits per sample (i.e., a total recording rate of $256 \mathrm{Mbit} \mathrm{s}^{-1}$ ).

Before, in the middle, and at the end of the phase referencing observations, we included "geodetic blocks", where we observed 18-21 bright sources from the International Celestial Reference Frame (ICRF) at $22 \mathrm{GHz}$ for $\sim 75 \mathrm{~min}$ to measure the tropospheric zenith delay offsets at each antenna (for a detailed discussion see Reid \& Brunthaler 2004; Brunthaler et al. 2005). The geodetic blocks were recorded with $8 \mathrm{IFs}$ of $8 \mathrm{MHz}$ in left circular polarization. The first IF was centered at a frequency of $22.01049 \mathrm{GHz}$, and the other seven IFs were separated by 12.5 , $37.5,100.0,262.5,312.5,412.5$, and $437.5 \mathrm{MHz}$, respectively. Here, only a single VLA antenna was used, which could observe only five IFs at $0.0,12.5,37.5,412.5$, and $437.5 \mathrm{MHz}$ relative to 22.01049 GHz.

The data were correlated at the VLBA Array Operations Center in Socorro, New Mexico. In the observation on 2008 May 03, the data were correlated at the position of known water maser emission $\left(09^{\mathrm{h}} 55^{\mathrm{m}} 51^{\mathrm{s}} .38702,+69^{\circ} 40^{\prime} 44^{\prime \prime}\right.$. 4676, J2000) with 128 spectral channels per IF and an integration time of $1 \mathrm{~s}$. The observation on 2009 April 08 was correlated at the position $09^{\mathrm{h}} 55^{\mathrm{m}} 51^{\mathrm{s}} .5500,+69^{\circ} 40^{\prime} 45^{\prime \prime} .792$, (J2000), close to SN 2008iz with 16 spectral channels and an integration time of $1 \mathrm{~s}$.

The data reduction was performed with the NRAO Astronomical Image Processing System (AIPS) in a standard manner. First we shifted the position of M 82 to the position of SN 2008iz using CLCOR in the observation on 2008 May 03. We applied the latest values of the Earth's orientation parameters and performed zenith delay corrections based on the results of the geodetic block observations. Total electron content maps of the ionosphere were used to correct for ionospheric phase changes. Amplitude calibration used system temperature measurements and standard gain curves. We performed a "manual phase-calibration" using the data from J1048+7143 to remove instrumental phase offsets among the frequency bands. Then, we fringe fitted the data from M $81^{*}$ and performed an amplitude self-calibration on M 81*. In the observation on 2008 May 03, we additionally first performed a phase-self calibration and later an amplitude self-calibration on SN 2008iz. In the observation on 2009 April 08, the inclusion of the phased VLA increased the noise in the images significantly, possibly due to a problem with the phasing of the array (the VLA was in B-configuration). Thus, we flagged the VLA data from this epoch.

\subsection{VLA observation on 2009 April 27 (1.4-43 GHz)}

We observed M 82 with the VLA on 2009 April 27 at 1.4, 4.8, $8.4,22$, and $43 \mathrm{GHz}$, for a total observing time of 4 hours. We observed with two frequency bands of $50 \mathrm{MHz}$, each in dual circular polarization. 3C 48 and $\mathrm{J} 1048+7143$ were used as primary flux density and phase calibrators, respectively. At 1.4, 4.8, and $8.4 \mathrm{GHz}$, we used a switching cycle of six minutes, spending one minute on the phase calibrator and five minutes on M 82. We repeated these cycles 5 times over the observations, yielding an integration time of $\sim 25 \mathrm{~min}$ at each frequency. At 22 and $43 \mathrm{GHz}$, we used a switching cycle of three minutes, spending one minute on the phase calibrator and two minutes on M 82. These cycles were repeated 10 times during the observation, yielding an integration time of $\sim 20 \mathrm{~min}$ at these frequencies.

The data reduction was performed in AIPS and involved amplitude calibration of $3 \mathrm{C} 48$ using source models, followed by a phase calibration using $\mathrm{J} 1048+7143$ and an additional phase and amplitude self-calibration on $\mathrm{J} 1048+7143$. The calibration was then transferred to the target source $M$ 82. We performed one phase-only self-calibration on M 82 at all frequencies, except $43 \mathrm{GHz}$ where the source is too weak.

\subsection{Chandra and Swift XRT observations}

The Chandra X-ray observatory (CXO) observed M 82 on 2008 October 4, and 2009 April 17 and 29. Each observation was taken with an exposure of about $18 \mathrm{ks}$. In these observations, the target was off the optical axis by more than 3 arcmin, where the point spread function resembles an extended ellipse covering multiple pixels.

M 82 was also observed with the Swift X-ray Telescope (XRT; Gehrels et al. 2004; Burrows et al. 2005) on 2007 January 26, 2008 May 1, and 2009 April 25 with exposures of $4.6 \mathrm{ks}$, $5.0 \mathrm{ks}$, and $4.7 \mathrm{ks}$, respectively. Due to the low resolution of the telescope, the collection of known X-ray sources appears as a single point-like source in the XRT data, so only count rates from the conglomerate of sources were measured. The data suffer from heavy pile-up, which is accounted for by extracting count rates from an annular region around the central, piled-up location $^{2}$.

\section{Results}

\subsection{VLBI images at $22 \mathrm{GHz}$}

We imaged the data of SN 2008iz from 2008 May 03 with a slightly super-resolved beam of $0.2 \times 0.2$ mas. The peak flux density was $5.9 \mathrm{mJy}$ beam $^{-1}$, the total emission was $32 \mathrm{mJy}$ and we achieved an image rms of $79 \mu \mathrm{Jy}_{\text {beam }^{-1}}$. The data from 2009 April 08 were imaged with natural weighting and a circular beam of $0.5 \times 0.5$ mas in order to have more sensitivity to extended emission. Here, the peak flux density was $0.36 \mathrm{mJy} \mathrm{beam}^{-1}$, the total emission was $4.3 \mathrm{mJy}$, and we achieved an image rms of $41 \mu \mathrm{Jy}^{\text {beam }^{-1}}$.

The supernova was clearly detected in both epochs (see Fig. 1). The source is already clearly resolved in the observation on 2008 May 03 and shows a ring-like structure, typical for a radio supernova. In the following eleven months the source expanded and faded significantly.

\section{2. $V L A$ radio spectrum}

M 82 was imaged at all frequencies using only data from baselines longer than $30 \mathrm{k} \lambda$ to ensure that most of the extended emission in M 82 is resolved out. Flux densities were extracted by fitting two-dimensional Gaussians to the images.

The measured flux densities of SN 2008iz and two other supernova remnants that could be easily separated from the diffuse background emission are listed in Table 1. The errors are estimated by adding in quadrature the formal error from the fit to

\footnotetext{
2 Given the pile-up and uncertainty in the XRT astrometry on the order of a few arcseconds, attempting to separate any supernova flux from the other flux is practically impossible.
} 
A. Brunthaler et al.: VLBI observations of SN 2008iz. I.

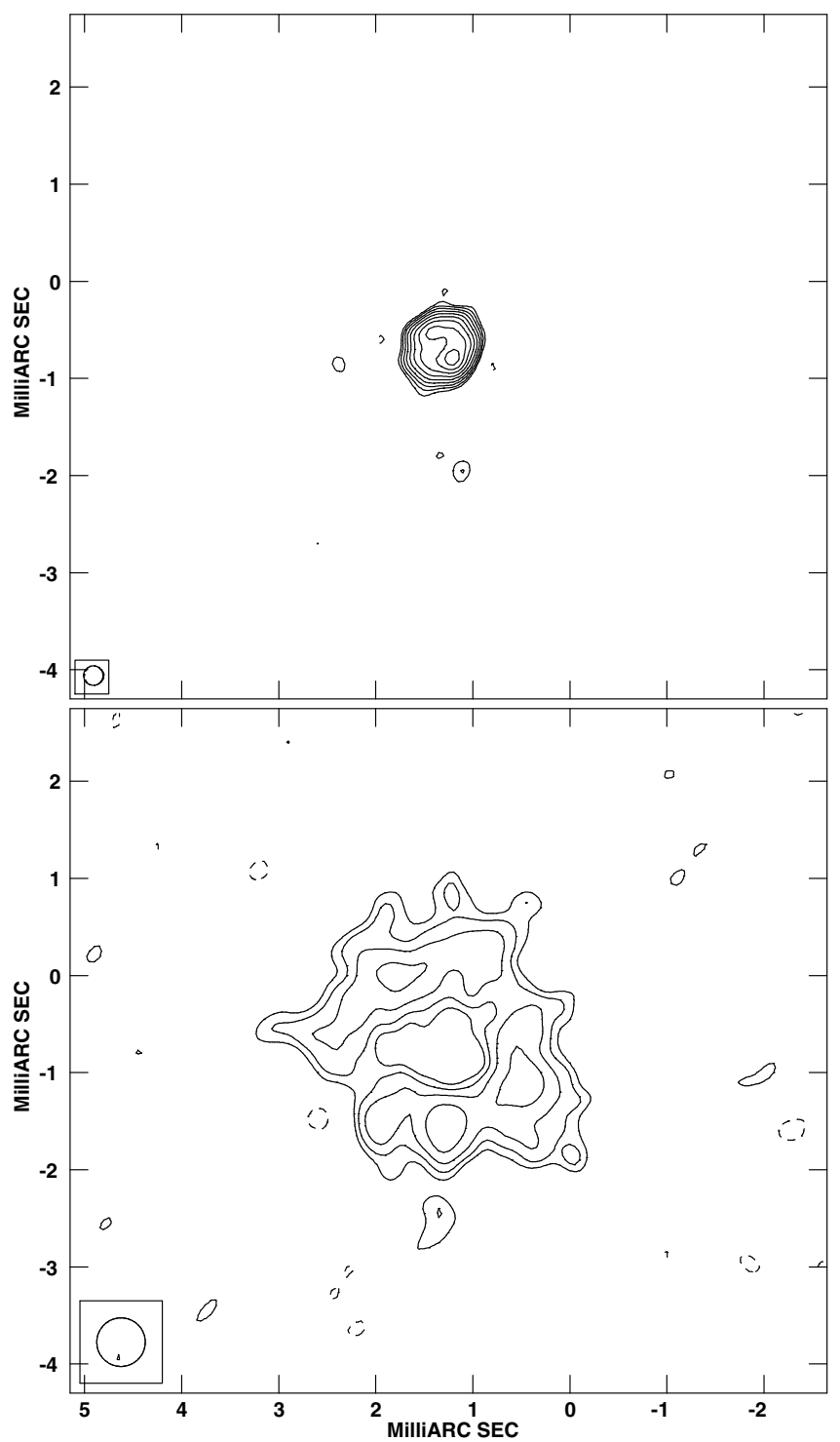

Fig. 1. HSA contour plots of SN2008iz at $22 \mathrm{GHz}$ in May 2008 (top) and April 2009 (bottom). Contours start at $0.3 \mathrm{mJy}$ (top), $0.1 \mathrm{mJy}$ (bottom) and increase with $\sqrt{2}$. The images are restored with a circular beam of FWHM 0.2 mas (top) and 0.5 mas (bottom). The original beam sizes were $0.31 \times 0.23$ mas at a position angle of $-34^{\circ}$ at the first epoch and $0.39 \times 0.27$ at a position angle of $-40^{\circ}$ at the second epoch.

Table 1. Flux densities of SN 2008iz and two other supernova remnants in M 82 from the VLA observation on 2009 April 27.

\begin{tabular}{cccccc}
\hline \hline Source & $F_{1.4 \mathrm{GHz}}$ & $F_{4.8 \mathrm{GHz}}$ & $F_{8.4 \mathrm{GHz}}$ & $F_{22 \mathrm{GHz}}$ & $F_{43 \mathrm{GHz}}$ \\
& {$[\mathrm{mJy}]$} & {$[\mathrm{mJy}]$} & {$[\mathrm{mJy}]$} & {$[\mathrm{mJy}]$} & {$[\mathrm{mJy}]$} \\
\hline
\end{tabular}

$\begin{array}{llllll}\text { SN 2008iz } & 55.3 \pm 6.0 & 29.5 \pm 4.5 & 13.7 \pm 2.5 & 6.2 \pm 0.7 & 2.5 \pm 0.4\end{array}$

$\begin{array}{lllllll}44.01+596 & - & 14.7 \pm 5.3 & 15.0 \pm 2.0 & 8.7 \pm 0.6 & 3.6 \pm 0.4\end{array}$

\begin{tabular}{llcccc}
$45.17+612-$ & - & $8.5 \pm 1.6$ & $5.8 \pm 0.7$ & $3.0 \pm 0.9$ & $0.8 \pm 0.3$ \\
\hline
\end{tabular}

the images, the difference between peak and integrated flux densities, and a 5\% error allowing for uncertainty in the overall flux density scale. Furthermore, we added an additional 5\% error at $1.4,4.8$, and $8.4 \mathrm{GHz}$, since we have more confusion from the extended emission at these frequencies. Note that the flux density at $4.8 \mathrm{GHz}$ is in good agreement with the flux densities obtained with MERLIN a few days later $(28.5 \pm 2 \mathrm{mJy})$ by Beswick et al. (2009).

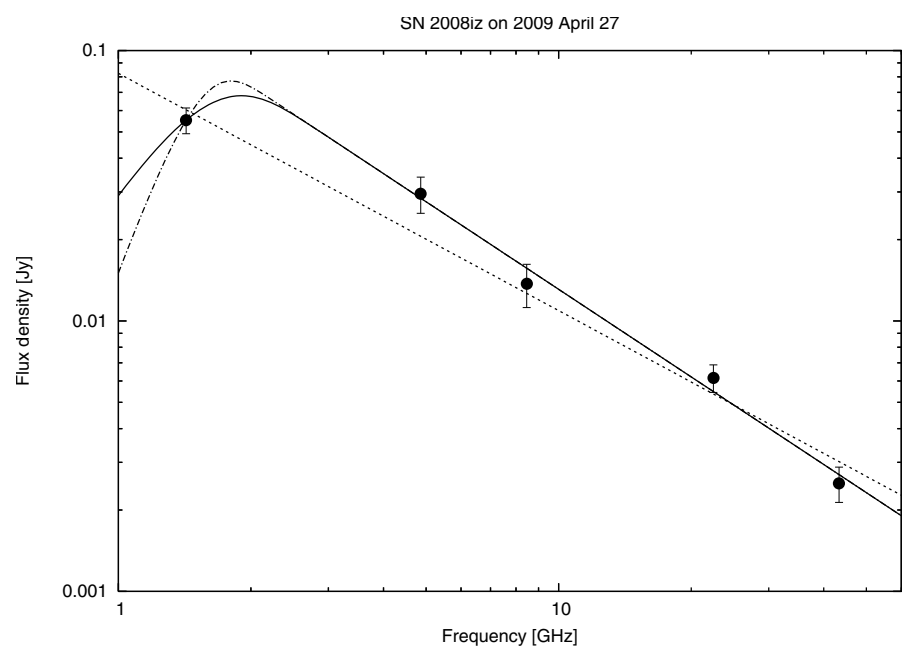

Fig. 2. Spectrum of SN 2008iz taken with the VLA on 2009 April 27. Also shown are a single power-law fit (dotted line) and two broken power-law fits with a spectral index of 2.5 (solid line) and 4.5 (dashdotted line) in the optically thick part.

The spectrum of SN 2008iz is shown in Fig. 2. First, we fitted a single power-law spectrum to the data, which resulted in a bestfit spectral index of $-0.88 \pm 0.07$. However, the fit has a large reduced $\chi^{2}$ value of 2.6 , due to the the $1.4 \mathrm{GHz}$ value being too low. Thus, to account for this, we also fitted the spectrum with a broken power-law,

$S(v)=S_{0}\left(\frac{v}{v_{0}}\right)^{\alpha}\left(1-\mathrm{e}^{-\left(\frac{v}{v_{0}}\right)^{\delta-\alpha}}\right)$

where $\alpha$ and $\delta$ are the spectral indices of the optically thin and thick parts of the spectrum, and $S_{0}$ and $v_{0}$ represent the maximum flux density and the peak frequency of the model spectrum. Since only one data point in the optically thick part of the spectrum is not enough to fit the spectral index in that region, we made two fits: one with a value for a synchrotron selfabsorbed spectrum $(\delta=2.5)$, and the other with a steeper freefree absorbed spectrum $(\delta=4.5)$. The reduced $\chi^{2}$ for both cases achieved a value of 0.9 . The fit (using $\delta=4.5$ ) yields a spectral index in the optically thin part of $\alpha=-1.08 \pm 0.08$ and a turnover frequency of $v_{0}=1.51 \pm 0.09 \mathrm{GHz}$. While the spectral index $\alpha$ is not affected by the choice of $\delta, v_{0}$ changes slightly to 1.55 for $\delta=2.5$. This indicates that the source was still optically thick and brightening at the lowest frequencies in April 2009 (for comparision: SN 1993J reached it's peak at $1.4 \mathrm{GHz} \sim 500$ days after the explosion; Weiler et al. 2002).

\subsection{X-ray upper limits}

The off-axis configuration and consequently the degraded angular resolution, as well as the diffuse background, significantly decreased the sensitivity of the Chandra measurements. The detection limit for these observations is estimated from the total emission around the radio position in a region with the same size as the point spread function. SN 2008iz is located close to several variable ultraluminous X-ray sources, but no emission was detected at the position of the supernova. Muxlow et al. (2009) and Muxlow et al. (2010) report the discovery of a second radio transient in M 82 with the MERLIN telescope. This source appeared between 2009 April 24 and 2009 May 5 and is located at a position with diffuse emission background. It is surrounded by a few point-like sources. There is no enhanced X-ray emission 
at the location of this second radio transient on 2009 April 17 or 2009 April 29.

In order to compare our results with those of SN 1993J, we assume that physical properties are similar at a similar ages, i.e., thermal bremsstrahlung emission with a temperature of $1.05 \mathrm{keV}$, abundances from Table 2, Col. 4 of Zimmermann \& Aschenbach (2003), and an absorption column density of $5.4 \times 10^{22} \mathrm{~cm}^{-2}$ (see Sect. 5.1). We then obtaing a $3 \sigma$ upper limit for SN 2008iz of $1.5 \times 10^{39} \mathrm{erg} \mathrm{s}^{-1}$ in the energy range $0.3-2.4 \mathrm{keV}$. This is consistent with the X-ray luminosity of SN 1993J at a similar age and energy range (Zimmermann \& Aschenbach 2003).

The $3 \sigma$ sensitivity at the location of the MERLIN transient, assuming a column density of $10^{22} \mathrm{~cm}^{-2}$ and a photon powerlaw index of 1.7 (an approximation to a thermal bremsstrahlung spectrum with a temperature of $10 \mathrm{keV}$, as seen in SN1995N; Fox et al. 2000), is found to be about $1.2 \times 10^{38} \mathrm{erg} \mathrm{s}^{-1}$ in $0.3-2.4 \mathrm{keV}$, which we take as the upper limit for the X-ray luminosity.

The resulting count rates in the Swift XRT data (0.5$8.0 \mathrm{keV})$ are $0.60 \pm 0.02,0.59 \pm 0.02$, and $0.68 \pm 0.02 \mathrm{~s}^{-1}$ on 2007 January 26, 2008 May 1, and 2009 April 25, respectively. There is no clear rise in total flux between the pre-SNe observation and the May 2008 post-explosion epoch at $\sim 75$ days, and, while a $\sim 15 \%$ increase in count rate is seen in the final observation, this is consistent with BeppoSAX observations of the central region of M 82 showing variations on the order of $15-30 \%$ (2-10 keV) on hour time-scales (Cappi et al. 1999). Indeed, such intrinsic variations are also seen over the course of each epoch in the XRT observations as well. Given the complications with the data and the intrinsic variability of the sources, strong constraints are difficult. However, since no increase in flux density larger than the intrinsic variability is seen, we can place an upper limit on the SNe X-ray flux of approximately $1.5 \times 10^{41} \mathrm{erg} \mathrm{s}^{-1}$ $(0.5-2.4 \mathrm{keV})$ on 2008 May 1 (assuming the same model as for the Chandra observations above).

\section{Expansion speed and explosion center}

\subsection{Estimates of size and position}

Estimating a physical size from the VLBI data is not straightforward, and different methods have been applied to determine the expansion curves of several radio supernovae (e.g. Bartel et al. 2002; Marcaide et al. 2009a,b). Estimating the source size in the image plane may have running biases (i.e., biases that depend on the source size) related to the different resolutions achieved in the different images (i.e., the structure is better resolved as the source expands). One way to avoid such biases is to use a $d y$ namic beam, i.e., a similar ratio between source size and beam size (e.g. Marcaide et al. 1997) at each epoch. Here we use several different methods to determine the source size. The results for each method are summarized in Table 2.

\subsubsection{Concentric rings}

First, we measured the intensity in concentric rings (of 0.05 mas width) using the AIPS task IRING. Examples of intensity profiles for both observations are shown in Fig. 3 together with fits to the data. One can define the radius, $R_{n} \%$, where the intensity falls to $n \%$ of the peak intensity, with any chosen value of $n$. Since $R_{n} \%$ can be multi-valued (i.e., the inner and outer radii), we always use the outer radius. Figure 3 shows the $R_{100 \%}$ (i.e. the peak itself) and $R_{50 \%}$ values. A value of $n=50 \%$ has the advantage that the profile is much steeper and the position can be

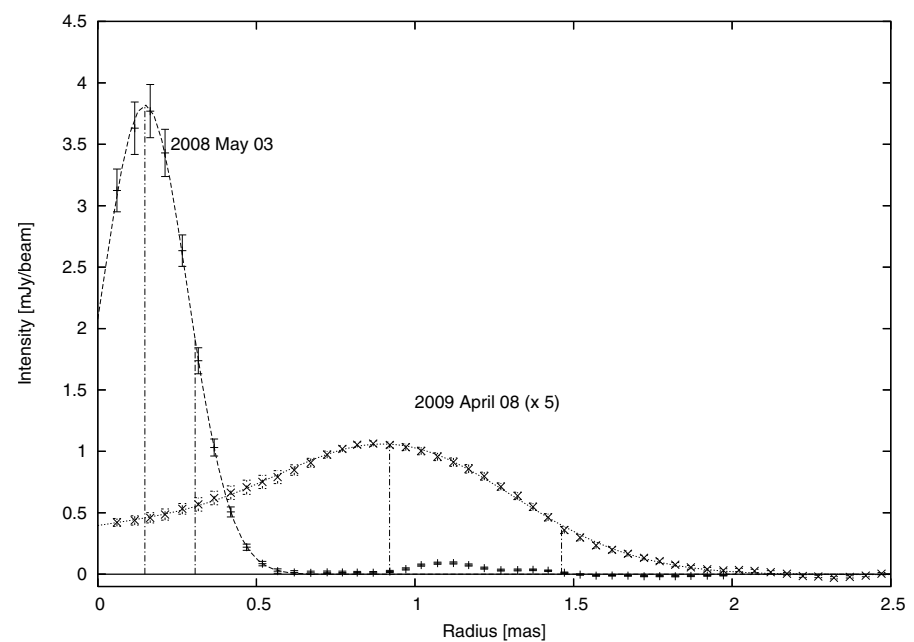

Fig. 3. Intensity of concentric rings of widths 0.05 mas in the images from 2008 May 03 and 2009 April 08. The flux densities in the second observation were scaled by a factor of 5 . The vertical lines mark the position of the peak and the radius where the intensity is $50 \%$ of the peak level.

better determined than at the peak (where the profile is flat). In order to avoid a running bias, it is important to use a similar ratio of source size to beam size (i.e. a dynamic beam) in each epoch. This method will give a size which is equal to the real size of the expanding shell times an unknown factor. This factor will be smaller for large values of $n$ (the measured sizes are smaller for large values of $n$ ). Figure 4 shows the images of SN 2008iz at both epochs and an image of the second epoch with a dynamic beam.

It is important to note that the intensity profile is sensitive to the position of the central pixel of the concentric rings. A position offset would smear out the intensity profile. This can be seen in Fig. 5 where we plot the width of the intensity profile for different position offsets in the first observation. As expected, the width shows a strong dependence on the position offset. We estimate the position of the center in the first epoch as $09^{\mathrm{h}} 55^{\mathrm{m}} 51^{\mathrm{s}} .55026,+69^{\circ} 40^{\prime} 45^{\prime \prime} .7913$ (J2000). Note that the uncertainty in the absolute position is dominated by the uncertainty of the position of the phase referencing source M $81 *$ ( $\sim 0.5$ mas).

\subsubsection{Common point method}

A different method to determine source size that uses the concept of a dynamic beam in a natural way is the Common-Point Method (CPM, Marcaide et al. 2009a). This method relies on the existence of a point in the radial profile of the supernova structure that remains unaltered under small changes in the convolving beam. This point is closely related to the source size (indeed, the ratio between the radial position of this point and the source radius is near unity; see Appendix A of Marcaide et al. 2009a, for details). In Fig. 6 we show the radial intensity profiles of SN 2008iz, computed from the azimuthal averages of the CLEAN models convolved using different beams. The "common points" in the profiles can be clearly seen in the figure.

\subsubsection{Model fitting}

Finally, we estimated the size from model-fitting to the visibilities, using a simplified model of the supernova radio structure. This method may have undetermined running biases if the model 

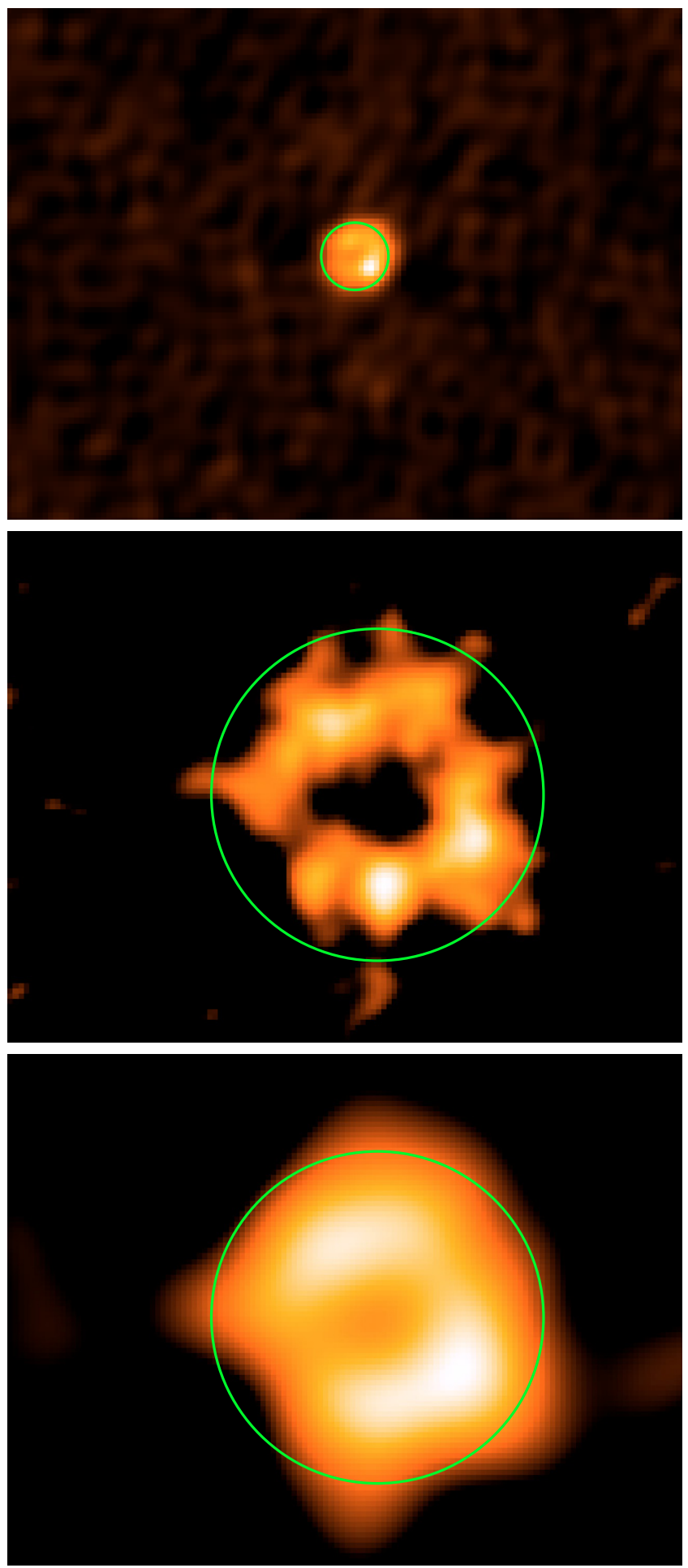

Fig. 4. HSA images at $22 \mathrm{GHz}$ of SN 2008iz in May 2008 (top) and April 2009 (middle and bottom). The bottom image is convolved with a dynamic beam of 0.99 mas (i.e. the ratios of beam size and source size are identical in the top and bottom images). The rings denote the best fitted positions and radii from CPM in both epochs, i.e. 320 and $1580 \mu$ as.

is not a good representation of the true supernova emission structure. We fitted the visibilities using a model of a spherical shell of $30 \%$ fractional width. This is the model that best describes the structure of SN 1993J in Marcaide et al. (2009a). A smaller fractional width between 20 and $25 \%$, as prefered by other authors

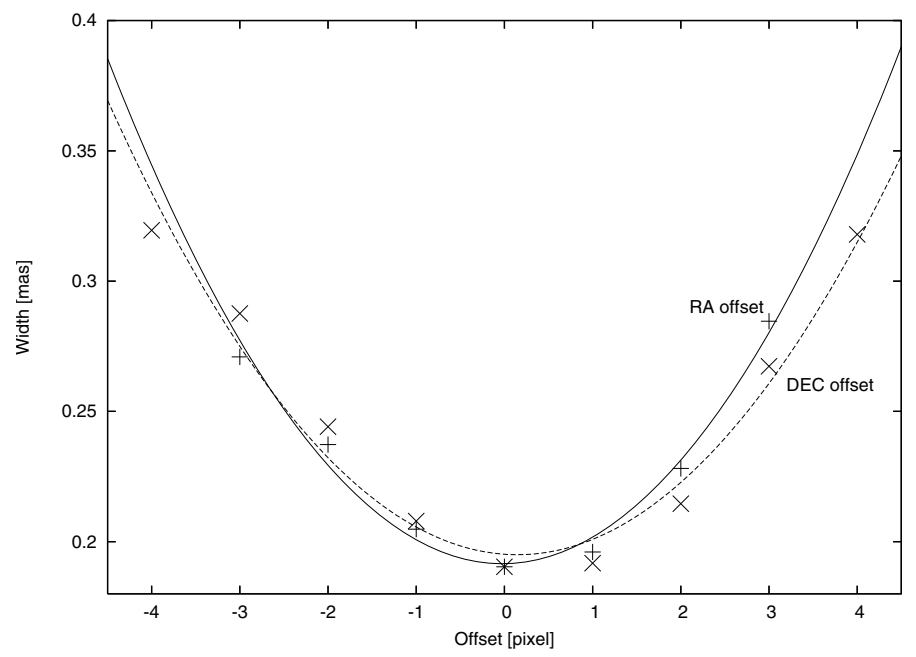

Fig. 5. The width of the intensity profile as a function of position offset (relative to a reference pixel) in the observation on 2008 May 03. One pixel corresponds to 0.05 mas. Shown are position offsets in right ascension $(+)$ and declination $(\times)$.

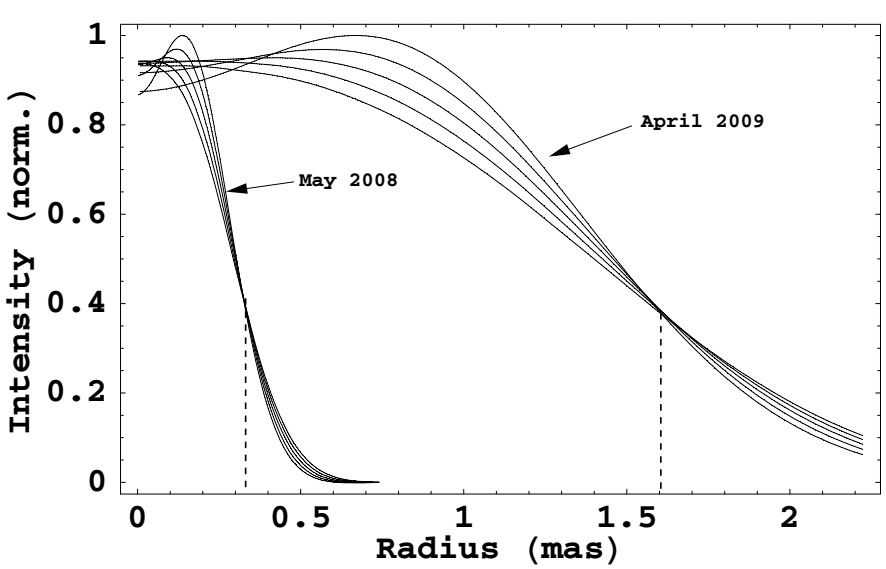

Fig. 6. Solid lines: radial intensity profiles of SN 2008iz at the two VLBI epochs, computed using different convolving beams (1.0, 1.1, 1.2, 1.3, and 1.4 times the source radius at each epoch). The radial positions of the common points at each epoch (see Sect. 4.1.2) are marked with dashed lines.

(Bartel et al. 2000, 2002; Bietenholz et al. 2003) or predicted by Chevalier (1982), could lead to different source sizes. However, the current data, based only on two epochs at one frequency, do not allow a relaible statement about the true fractional width of SN2008iz. For the model-fitting, we used a modified version of the subroutine MODELFIT in the program DIFMAP (Shepherd et al. 1995), which fits a parameterized shell model to the real and imaginary parts of the visibilities. The use of the real and imaginary parts of the visibilities (instead of amplitudes and phases) is more robust from a statistical point of view, especially in the case of low signal-to-noise data, since the noise involved in the data is gaussian-like (as assumed in the modelling algorithms based on a $\chi^{2}$ minimization).

\subsection{Expansion speed}

Using the $R_{50 \%}$ value for the physical size, we obtain a radius of $307 \mu$ as on 2008 May 03 and $1462 \mu$ as on 2009 April 08. The measured sizes correspond to an average expansion speed of $1243 \mu$ as $\mathrm{yr}^{-1}$ or $v_{\exp }=21200 \mathrm{~km} \mathrm{~s}^{-1}$. Extrapolating this 
Table 2. Source sizes, expansion speeds, a lower limit for the expansion index $m$, and explosion dates (in 2008).

\begin{tabular}{lcccccc}
\hline \hline Method $^{a}$ & $\begin{array}{c}R_{2008} \\
{[\mu \mathrm{as}]}\end{array}$ & $\begin{array}{c}R_{2009} \\
{[\mu \mathrm{as}]}\end{array}$ & $\begin{array}{c}v_{\text {exp }, m=1} \\
\mathrm{~km} \mathrm{~s}^{-1}\end{array}$ & $\begin{array}{c}t_{0} \\
(m=1)\end{array}$ & $m$ & $\begin{array}{c}t_{0} \\
(m=0.89)\end{array}$ \\
\hline$R_{100 \%}$ & 149 & 1008 & 15800 & Mar. 05 & $>0.86$ & Mar. 19 \\
$R_{50 \%}$ & 307 & 1462 & 21200 & Feb. 02 & $>0.70$ & Feb. 22 \\
$R_{25 \%}$ & 380 & 1676 & 23800 & Jan. 24 & $>0.66$ & Feb. 14 \\
CPM & 320 & 1580 & 23100 & Feb. 07 & $>0.71$ & Feb. 25 \\
MODELFIT & 320 & 1390 & 19600 & Jan. 22 & $>0.65$ & Feb. 12 \\
\hline
\end{tabular}

Notes. ${ }^{(a)}$ For a description of the different methods see Sect. 4.1.

expansion back to a radius of zero and assuming constant expansion $\left(r \propto t^{m}\right.$ with an expansion index $\left.m=1\right)$ leads to an explosion date $t_{0}$ of 2008 February 02. However, a significant deceleration (i.e. $m<1$ ) would shift the explosion date to a later time. Since the supernova was first seen in a VLA observation on 2008 March 24, one can use this to give a lower limit for the expansion index $m$ of 0.7. Marchili et al. (2010) model the $5 \mathrm{GHz}$ light curve of SN 2008iz and find evidence of a modest deceleration with an expansion index $m=0.89$ and an explosion date of 2008 February 18 ( \pm 6 days). Extrapolating the expansion seen on the VLBI images backward using this expansion index, yields an explosion date of 2008 February 22. This value for the explosion date is in reasonable agreement with the estimate from the $5 \mathrm{GHz}$ light curve.

The resulting source sizes for the different methods are summarized in Table 2. Most methods give similar expansion speeds between 19600 and $23800 \mathrm{~km} \mathrm{~s}^{-1}$. Since the $R_{100 \%}$ values give a significantly lower expansion speed and a very late explosion date, we conclude that the $R_{100 \%}$ values underestimate the true source sizes significantly. Hence, we do not consider these values in the following analysis. Based on the information of the two VLBI images, we conclude that the explosion occured between 2008 January 22 and 2008 March 24. We get a lower limit for the expansion index of $m>0.65$, and an average expansion speed in the range of 19600-23800 $\mathrm{km} \mathrm{s}^{-1}$.

Since the CPM source sizes should be very close to the real sizes, the expansion speed is very close to the average value of all methods $\left(\sim 22000 \mathrm{~km} \mathrm{~s}^{-1}\right.$, excluding $R_{100 \%}$ as discussed above), and the explosion date is in very good agreement with the light curve modeling, we adopt these values. The formal errors in our various fits are exceedingly small (of the order of 20 $\mu$ as). Since the systematic errors from the different methods are much larger, the spread in velocities $\left( \pm 1650 \mathrm{~km} \mathrm{~s}^{-1}\right)$ and explosion dates ( \pm 7 days) better reflects our uncertainties.

This expansion speed is much higher than the values reported in Brunthaler et al. (2009b). There are three reasons for this discrepancy. Firstly, the lower value was based on a preliminary data reduction, without the data from the phased VLA and without the geodetic block corrections. Secondly, the radius of the brightest emission was used as an approximation for the source size. However, as shown here, this method underestimates the true expansion speed. Thirdly, the sizes were measured by hand from the images and this gave an overestimated size in the first epoch, where the source was still very compact.

\subsection{Expansion center, self-similarity, and anisotropic expansion}

The ring is not symmetric and the brightest region in both images is the south-western part of the shell. This could be caused
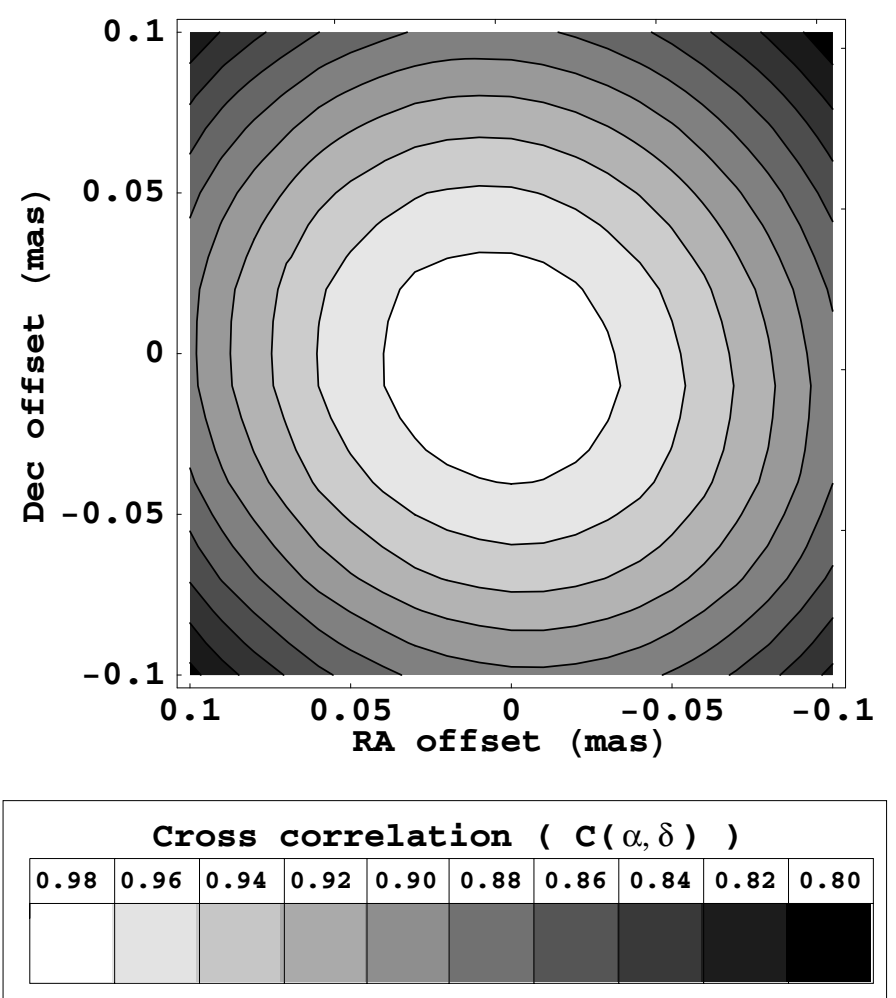

Fig. 7. Cross-correlation coefficient, $C(\alpha, \delta)$, between the images of the two VLBI epochs (scaling the first epoch to the size of the second), as a function of the coordinates of the explosion center, $(\alpha, \delta)$, taking as reference the position of the maximum $C(\alpha, \delta)$ (see Sect. 4.3).

either by an asymmetry in the explosion or in the circumstellar medium (CSM), i.e. a clump with higher density in the southwest. A clumpy CSM is very common for red supergiant stars (e.g. Smith et al. 2009) which are likely progenitors for this supernova. Furthermore, a comparison of the $5 \mathrm{GHz}$ light curve and the available $22 \mathrm{GHz}$ data shows evidence for a clumpy CSM (Marchili et al. 2010).

As mentioned in Sect. 4.1.1, we also estimated the central position of the ring. We do not detect any significant shift in the position, with an upper limit of $\sim 50 \mu$ as in right ascension and $100 \mu$ as in declination. To verify the relative astrometric accuracy of the positions, we also imaged the three extragalactic background sources 0945+6924, 0948+6848, and 1004+6936. The average position change between the two observations of M 81* relative to these quasars is $1 \pm 15 \mu$ as in right ascension and $22 \pm 53 \mu$ as in declination, where the uncertainties are the standard deviation ${ }^{3}$. Thus, we can rule out that any significant position shift is introduced by jet motion in M $81 *$. We note that the angular separation of M 82 to M $81 *$ is not larger than the angular separation between M $81^{*}$ and the three background quasars. Hence, systematic errors in the astrometry should be similar for all sources.

According to Chevalier (1982), the expansion of a supernova is self-similar, i.e., the source structure remains unaltered regardless of the source size. This model of a self-similar expansion has been extensively tested with SN 1993J (e.g. Marcaide et al. 1997, 2009a; Bartel et al. 2002). Although some deviations from self-similarity were found in the expansion of SN 1993J, this supernova kept its structure nearly self-similar for more than a

\footnotetext{
3 These values give $1 \sigma$ upper limits on the proper motion of M 81 of $260 \mathrm{~km} \mathrm{~s}^{-1}$ in right ascension and $1270 \mathrm{~km} \mathrm{~s}^{-1}$ in declination.
} 
decade. If the expansion of SN 2008iz was also self-similar between our two VLBI epochs, the VLBI images obtained should be equal (regardless of the global flux density scale) if we scale the first image to the size of the second one and use the same convolving beam for both images. However, the result of scaling the first VLBI image, to compare to the second one, depends on the point in the image that is chosen as the center of expansion. Therefore, a direct comparison between the images, to check the self-similarity in the expansion, is not possible if the coordinates of the expansion center of the supernova are not known.

In order to determine the coordinates of the expansion center of the supernova independently from the method described above, we computed different scaled versions of the image of the first VLBI epoch using different centers of expansion. The scaled images were obtained by scaling the positions of the CLEAN components of the model, according to the ratios of the supernova sizes reported in the previous section. We then used the same beam to convolve the CLEAN model of the second epoch and the resulting (scaled) CLEAN models of the first epoch. Finally, we compared the resulting image of the second epoch with the resulting (scaled) images of the first epoch by computing the cross correlation between both images:

$C(\alpha, \delta)=\frac{\sum_{i} I_{2}^{i} \times I_{1}^{i}(\alpha, \delta)}{\sqrt{\sum_{i}\left(I_{1}^{i}(\alpha, \delta)\right)^{2} \times \sum_{i}\left(I_{2}^{i}\right)^{2}}}$

where $I_{2}^{i}$ is the $i$ th pixel of the image of the second epoch and $I_{1}^{i}(\alpha, \delta)$ is the $i$ th pixel of the scaled version of the image of the first epoch, taking the point $(\alpha, \delta)$ as the expansion center. Assuming a self-similar expansion, the coordinates of the maximum value of $C(\alpha, \delta)$ are an estimate of the position of expansion center of the supernova, which gives us the coordinates of the explosion. In Fig. 7 we show the cross correlation of the images computed for different expansion centers, $(\alpha, \delta)$, expanding the image of the first epoch according to the size estimated with the CPM. The maximum correlation takes place at the coordinates $\alpha=09^{\mathrm{h}} 55^{\mathrm{m}} 51^{\mathrm{s}} .55025$ and $\delta=+69^{\circ} 40^{\prime} 45^{\prime \prime} .79133$. These coordinates do not change by more than $10 \mu$ as if we use, instead, the size estimated with model-fitting to expand the image of the first VLBI epoch. This position agrees with the position given in Sect. 4.1.1 to within $60 \mu$ as and verifies that the position of the expansion center has not changed between the two epochs.

Additionally, the value of $C(\alpha, \delta)$ at its maximum is a measure of the degree of self-similarity (and/or anisotropy) in the expansion. The maximum cross-correlation of the images is 0.98 , if we use the CPM size to expand the image of the first epoch, and 0.97, if we use instead the model-fitting size. These values are very close to 1 , which is the case of a perfect self-similar expansion. Therefore, we conclude that the expansion of SN 2008iz was self-similar to a high degree between our two VLBI epochs.

\section{Discussion}

\subsection{Column density and extinction}

The non-detection of SN 2008iz in the optical, infrared, and $\mathrm{X}$-rays indicates that it exploded inside or behind a very dense cloud. Indeed, the ${ }^{12} \mathrm{CO}(J=2 \rightarrow 1)$ line intensity map in Weiß et al. (2001) shows a prominent cloud exactly at the position of the supernova (Fig. 8). The line intensity at the position of SN 2008iz is $\sim 1800 \mathrm{~K} \mathrm{~km} \mathrm{~s}^{-1}$. Using the Galactic conversion factor $X_{\mathrm{CO}}=1.6 \times 10^{20} \mathrm{~cm}^{-2}\left(\mathrm{~K} \mathrm{~km} \mathrm{~s}^{-1}\right)^{-1}$, this corresponds to

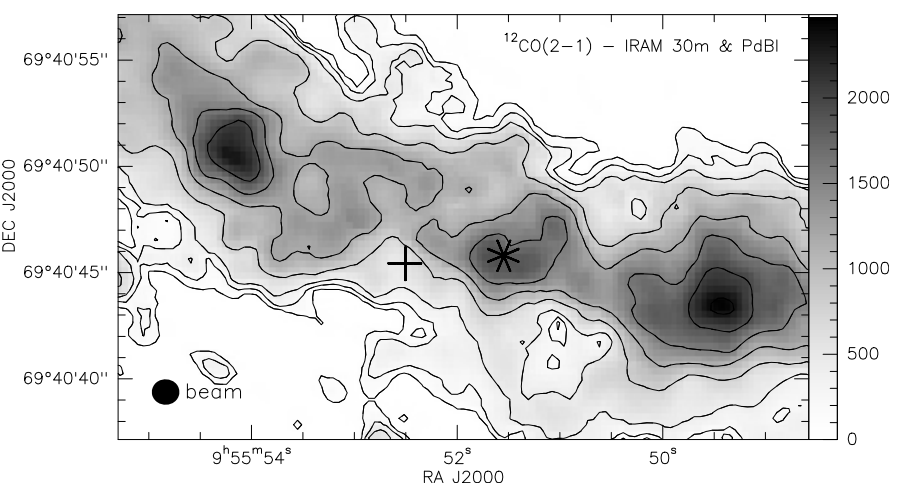

Fig. 8. ${ }^{12} \mathrm{CO}(J=2 \rightarrow 1)$ intensity map of the central region of M 82 from Weiß et al. (2001) with the positions of SN 2008iz (star) and the MERLIN transient (cross). The contours correspond to 200, 400, 600, $800,12001600,2000$, and $2400 \mathrm{~K} \mathrm{kms}^{-1}$. The resolution of the observation is $1.5^{\prime \prime} \times 1.4^{\prime \prime}(\sim 25 \mathrm{pc})$.

a $\mathrm{H}_{2}$ column density $N\left(\mathrm{H}_{2}\right)$ of $\sim 29 \times 10^{22} \mathrm{~cm}^{-2}$. However, Weiß et al. (2001) find much smaller and spatially variable conversion factors from radiative transfer calculations, that lead to smaller $\mathrm{H}_{2}$ column densities. At the position of SN 2008iz, their conversion factor is $\sim 0.3 \times 10^{20} \mathrm{~cm}^{-2}\left(\mathrm{~K} \mathrm{~km} \mathrm{~s}^{-1}\right)^{-1}$ (their Fig. 10). This leads to an $\mathrm{H}_{2}$ column density of $\sim 5.4 \times 10^{22} \mathrm{~cm}^{-2}$. However, the $\mathrm{CO}$ observations were performed with a linear resolution of $\sim 25 \mathrm{pc}$. Thus, it is possible that the supernova is located behind a smaller cloud with much higher column density. We also note that the ${ }^{12} \mathrm{CO}$ line intensity at the position of the MERLIN transient is almost 4 times smaller $\left(\sim 500 \mathrm{~K} \mathrm{~km} \mathrm{~s}^{-1}\right)$.

Taking the latter value $\left(5.4 \times 10^{22} \mathrm{~cm}^{-2}\right)$ for $N\left(\mathrm{H}_{2}\right)$ and assuming that all of the column is between SN 2008iz and us, we derive a visual extinction, $A_{\mathrm{V}}$, of $24.4 \mathrm{mag}$. Here we have used the relation between optical extinction and atomic hydrogen column density, $N(\mathrm{H})$, derived by Güver \& Özel (2009) from X-ray absorption data of a sample of Galactic SNRs, and assumed that all hydrogen is in molecular form, i.e., $N\left(\mathrm{H}_{2}\right)=2 N(\mathrm{H})$. Such a high value of the extinction would explain the lack of an optical counterpart.

The derived extinction is much higher than toward SN 1993J, for which Richmond et al. (1994) discuss values of $A_{\mathrm{V}}=0.7$ and 1.0 mag. With the extinction law given by Cardelli et al. (1989), $A_{K}=0.114 A_{\mathrm{V}}$, we calculate $2.8 \mathrm{mag}$ for the $K$-band $(2.2 \mu \mathrm{m})$ extinction. Fraser et al. (2009) report an even higher $K$-band extinction of up to $11 \mathrm{mag}$ based on their non-detection ( $3 \sigma$ upper limit on the absolute $K$-band magnitude of -5 mag) and the assumption that the infrared light curve of SN 2008iz behaves similarly to that of SN 1993J. Thus,

SN 2008iz is either very weak in the infrared, compared to SN 1993J, or behind a smaller but even denser cloud than estimated here.

\subsection{Expansion velocity and synchrotron self-absorption}

Chevalier (1998) proposed a way to estimate the mean expansion velocity of a supernova based on the radio-light curve and assuming that synchrotron self-absorption (SSA) dominates the light curve at the peak. If SSA is not the dominant absorption process at the peak of the light curve, then the estimate of the expansion velocity is a lower bound to the real expansion velocity of the radio shell. Following Chevalier (1998), the estimate mean expansion velocity at the peak of the radio-light curve 
Table 3. Comparison between SN 1993J and SN 2008iz.

\begin{tabular}{lccc}
\hline \hline Property & & SN 1993J & SN2008iz \\
\hline spectral index $\alpha$ & & $-0.99^{a}$ & $-1.08^{b}$ \\
$L_{5 \mathrm{GHz}}$ & {$\left[10^{27} \mathrm{erg} \mathrm{s}^{-1} \mathrm{~Hz}^{-1}\right]$} & $1.5^{c}$ & $2.5^{d}$ \\
$t_{\text {peak }}-\mathrm{t}_{0}$ & {$[\mathrm{days}]$} & $180^{c}$ & $\sim 120^{d}$ \\
$v_{\text {VLBI }}$ & {$\left[\mathrm{km} \mathrm{s}^{-1}\right]$} & $14900^{e}$ & $21200^{b}$ \\
$L_{\mathrm{X}-\text { ray att } \sim 220 \text { days }}$ & {$\left[10^{38} \mathrm{erg} \mathrm{s}^{-1}\right]$} & $\sim 8^{f}$ & $<15^{b}$ \\
\hline
\end{tabular}

Notes. References: ${ }^{(a)}$ van Dyk et al. (1994); ${ }^{(b)}$ this work; ${ }^{(c)}$ Weiler et al. (1998) ; ${ }^{(d)}$ Marchili et al. (2010) ; ${ }^{(e)}$ Marcaide et al. (1995); (f) Zimmermann \& Aschenbach (2003).

(assuming dominant SSA) is given by the following equation:

$$
\begin{aligned}
& V_{\mathrm{SSA}}\left(\mathrm{km} \mathrm{s}^{-1}\right)= 5.3786 \times 10^{6}(\beta \phi)^{-0.053}\left(\frac{F_{\mathrm{p}}}{1 \mathrm{Jy}}\right)^{0.47} \\
& \times\left(\frac{D}{1 \mathrm{Mpc}}\right)^{0.95}\left(\frac{v}{1.0 \mathrm{GHz}}\right)^{-1}\left(\frac{t_{\mathrm{p}}}{1 \text { day }}\right)^{-1},
\end{aligned}
$$

where $\beta$ is the ratio of the relativistic particle energy density to the magnetic field energy density (if we assume energy equipartition, $\beta=\frac{4}{3(1+k)}$, where $k$ ranges from 1 to 2000, see Pacholczyk 1970 , Chap. 7), $\phi$ is the filling factor of the emitting region (we assume a shell of width equal to $30 \%$ of the outer radius, which yields $\phi=0.66), F_{\mathrm{p}}$ is the flux density at the peak, $D$ is the distance, $v$ is the observing frequency, $t_{\mathrm{p}}$ is the supernova age at the peak, and we use a spectral index $\alpha=-1$ (see Eqs. (11) and (13) of Chevalier 1998).

For SN2008iz, Eq. (3) yields a mean expansion velocity (depending on $\mathrm{k}$ ) in the range $8100-11800 \mathrm{~km} \mathrm{~s}^{-1}$ at the peak of the $5 \mathrm{GHz}$ radio light curve. Using an expansion index of 0.89 , these velocities translate into a mean expansion velocity in the range $7000-10300 \mathrm{~km} \mathrm{~s}^{-1}$ on 2009 April 27. This range of velocities is a factor $\sim 2$ smaller than the velocities estimated from our VLBI observations, thus indicating that, in contrast to the case of SN1993J, SSA may not be the dominant absorption mechanism in the SN2008iz radio emission. This is also consistent with the results in Marchili et al. (2010) who were able to model the radio light curve of SN2008iz assuming that SSA effects are much smaller than free-free absorption (FFA) during the whole supernova expansion.

\subsection{Comparison with other type II radio supernovae}

Estimates of the expansion velocities of other type II radio supernovae have been estimated from VLBI observations, and very different results have been obtained. For instance, the mean expansion velocity of SN 1979C during the first year after explosion has been estimated to be $\sim 10000-11000 \mathrm{~km} \mathrm{~s}^{-1}$ (Bartel \& Bietenholz 2003; Marcaide et al. 2009b); for SN 1986J, a velocity of $\sim 14700 \mathrm{~km} \mathrm{~s}^{-1}$ was obtained by Pérez-Torres et al. (2002b), while Bietenholz et al. (2002) find $20000 \mathrm{~km} \mathrm{~s}^{-1}$ 3 month after the explosion; Staveley-Smith et al. (1993) report a mean expansion speed of $\sim 35000 \mathrm{~km} \mathrm{~s}^{-1}$ during the first years for SN 1987A before it slowed down to $\sim 4800 \mathrm{~km} \mathrm{~s}^{-1}$; for SN 2004et, the expansion velocity was $>15700 \mathrm{~km} \mathrm{~s}^{-1}$ (MartíVidal et al. 2007); and for SN 2008ax, an expansion velocity as large as $52000 \mathrm{~km} \mathrm{~s}^{-1}$ was obtained (Martí-Vidal et al. 2009). Estimates of the expansion velocities of other supernova remnants in M 82 (the host galaxy of SN 2008iz) have also been reported to range between $\sim 1500$ and $11000 \mathrm{~km} \mathrm{~s}^{-1}$ (Beswick et al. 2006). The larger velocities above are much higher (by a factor of 3-22) than the predicted velocities from the model of Chevalier \& Fransson (2001), based on the high pressure expected in the interstellar medium (ISM) of M 82. The expansion velocity reported in this paper for SN2008iz is indeed a factor $\sim 40$ larger than the predicted velocities in Chevalier \& Fransson (2001), although of the same order-of-magnitude than the velocities reported in Beswick et al. (2006) for the other remnants in M 82, and the typical velocities of the other type II supernovae observed to date.

Weiler et al. (1998) find a correlation between peak radio luminosity at $5 \mathrm{GHz}$ and the time between the explosion and the peak in the $5 \mathrm{GHz}$ light curve for type II supernovae. The $5 \mathrm{GHz}$ light curve of SN 2008iz from Marchili et al. (2010) gives a peak luminosity of $\sim 2.5 \times 10^{27} \mathrm{erg} \mathrm{s}^{-1} \mathrm{~Hz}^{-1}$ at $\sim 120$ days after the explosion. These values are well within the scatter of the correlation. Thus it seems plausible that SN 2008iz is also a type II supernova.

Since SN 2008iz and SN 1993J are located at very similar distances, this allows a detailed comparison between these two supernovae. Several properties of both supernovae are summarized in Table 3. The radio spectral indices, the peak radio luminosities, rise times, and early VLBI expansion velocities are similar (considering that the rise times and peak radio luminosities can vary by several orders of magnitudes for type II radio supernovae). The non-detection in X-rays of SN 2008iz can be attributed to absorption by the dense molecular cloud seen in the $\mathrm{CO}$ data.

\section{Summary}

In this paper we presented the first VLBI images, a VLA radio spectrum from 1.4 to $43 \mathrm{GHz}$, and Chandra X-ray observations of SN 2008iz. Our main results are as follows:

- The VLBI images separated by $\sim 11$ month show a shell-like structure expanding with a velocity of $\sim 23000 \mathrm{~km} \mathrm{~s}^{-1}$.

- The inferred expansion speed is a factor of 2 higher than expected if SSA dominates the light curve, suggesting that SSA is not important for the radio emission.

- The most likely explosion date is in mid-February 2008, but not earlier than January 22 and not later than March 24.

- We find a high degree of self-similarity between the two VLBI observations and no evidence for an asymmetric explosion.

- The VLA radio spectrum is well fitted by a broken power law with a turnover frequency of $1.5 \pm 0.1 \mathrm{GHz}$ and a spectral index of $-1.08 \pm 0.08$ in the optically thin part.

- SN 2008iz is located behind (or inside) a large molecular cloud with an $\mathrm{H}_{2}$ column density of $5.4 \times 10^{22} \mathrm{~cm}^{-2}$ (on a scale of $25 \mathrm{pc}$ ), corresponding to a visual extinction, $A_{\mathrm{V}}$, of $24.4 \mathrm{mag}$.

- Due to the high column density, we obtain only upper limits on the X-ray luminosity of $1.5 \times 10^{41}$ and $1.5 \times 10^{39} \mathrm{erg} \mathrm{s}^{-1}$ $\sim 75$ and $\sim 200$ days after the explosion, which are consistent with the X-ray luminosities of SN 1993J at similar ages. We also obtain an upper limit for the X-ray luminosity of the second radio transient of $1.2 \times 10^{38} \mathrm{erg} \mathrm{s}^{-1}$ (in $0.3-2.4 \mathrm{keV}$ ).

Acknowledgements. This work is partially based on observations with the 100-m telescope of the MPIfR (Max-Planck-Institut für Radioastronomie) at Effelsberg. MV is a fellow of the Alexander von Humboldt Foundation in Germany. 


\section{References}

Bartel, N., \& Bietenholz, M. F. 2003, ApJ, 591, 301

Bartel, N., Bietenholz, M. F., Rupen, M. P., et al. 2000, Science, 287, 112

Bartel, N., Bietenholz, M. F., Rupen, M. P., et al. 2002, ApJ, 581, 404

Bartel, N., Bietenholz, M. F., Rupen, M. P., \& Dwarkadas, V. V. 2007, ApJ, 668, 924

Beswick, R. J., Riley, J. D., Marti-Vidal, I., et al. 2006, MNRAS, 369, 1221

Beswick, R. J., Muxlow, T. W. B., Pedlar, A., et al. 2009, The Astronomer's Telegram, 2060, 1

Bietenholz, M. F., Bartel, N., \& Rupen, M. P. 2001, ApJ, 557, 770

Bietenholz, M. F., Bartel, N., \& Rupen, M. P. 2002, ApJ, 581, 1132

Bietenholz, M. F., Bartel, N., \& Rupen, M. P. 2003, ApJ, 597, 374

Brunthaler, A., Menten, K. M., Reid, M. J., et al. 2009a, A\&A, 499, L17

Brunthaler, A., Menten, K. M., Reid, M. J., et al. 2009b, Central Bureau Electronic Telegrams, 1803, 1

Brunthaler, A., Reid, M. J., \& Falcke, H. 2005, in Future Directions in High Resolution Astronomy, ASP Conf. Ser., 340, 455

Burrows, D. N., Hill, J. E., Nousek, J. A., et al. 2005, Space Sci. Rev., 120, 165

Cappi, M., Palumbo, G. G. C., Pellegrini, S., \& Persic, M. 1999, Astron. Nachr., 320,248

Cardelli, J. A., Clayton, G. C., \& Mathis, J. S. 1989, ApJ, 345, 245

Chevalier, R. A. 1982, ApJ, 258, 790

Chevalier, R. A. 1998, ApJ, 499, 810

Chevalier, R. A., \& Fransson, C. 2001, ApJ, 558, L27

Fox, D. W., Lewin, W. H. G., Fabian, A., et al. 2000, MNRAS, 319, 1154

Fraser, M., Smartt, S. J., Crockett, M., et al. 2009, The Astronomer's Telegram, 2131, 1

Freedman, W. L., Hughes, S. M., Madore, B. F., et al. 1994, ApJ, 427, 628

Gehrels, N., Chincarini, G., Giommi, P., et al. 2004, ApJ, 611, 1005

Güver, T., \& Özel, F. 2009, MNRAS, 400, 2050

Marcaide, J. M., Alberdi, A., Ros, E., et al. 1995, Science, 270, 1475
Marcaide, J. M., Alberdi, A., Ros, E., et al. 1997, ApJ, 486, L31

Marcaide, J. M., Martí-Vidal, I., Alberdi, A., et al. 2009a, A\&A, 505, 927

Marcaide, J. M., Martí-Vidal, I., Perez-Torres, M. A., et al. 2009b, A\&A, 503, 869

Marchili, N., Marti-Vidal, I., Brunthaler, A., et al. 2010, A\&A, 509, A47

Martí-Vidal, I., Marcaide, J. M., Alberdi, A., et al. 2007, A\&A, 470, 1071

Martí-Vidal, I., Marcaide, J. M., Alberdi, A., et al. 2009, A\&A, 499, 649

Muxlow, T. W. B., Beswick, R. J., Pedlar, A., et al. 2009, The Astronomer's Telegram, 2073, 1

Muxlow, T. W. B., Beswick, R. J., Garrington, S. T., et al. 2010, MNRAS, 404, L109

Pacholczyk, A. G. (ed.) 1970, Radio astrophysics. Nonthermal processes in galactic and extragalactic sources

Pérez-Torres, M. A., Alberdi, A., \& Marcaide, J. M. 2001, A\&A, 374, 997

Pérez-Torres, M. A., Alberdi, A., \& Marcaide, J. M. 2002a, A\&A, 394, 71

Pérez-Torres, M. A., Alberdi, A., Marcaide, J. M., et al. 2002b, MNRAS, 335, L23

Reid, M. J., \& Brunthaler, A. 2004, ApJ, 616, 872

Richmond, M. W., Treffers, R. R., Filippenko, A. V., et al. 1994, AJ, 107, 1022

Shepherd, M. C., Pearson, T. J., \& Taylor, G. B. 1995, in BAAS, ed. B. J. Butler, \& D. O. Muhleman, 27, 903

Smith, N., Hinkle, K. H., \& Ryde, N. 2009, AJ, 137, 3558

Staveley-Smith, L., Briggs, D. S., Rowe, A. C. H., et al. 1993, Nature, 366, 136

van Dyk, S. D., Weiler, K. W., Sramek, R. A., Rupen, M. P., \& Panagia, N. 1994, ApJ, 432, L115

Weiler, K. W., van Dyk, S. D., Montes, M. J., Panagia, N., \& Sramek, R. A. 1998, ApJ, 500, 51

Weiler, K. W., Panagia, N., Montes, M. J., \& Sramek, R. A. 2002, ARA\&A, 40, 387

Weiß, A., Neininger, N., Hüttemeister, S., \& Klein, U. 2001, A\&A, 365, 571

Zimmermann, H., \& Aschenbach, B. 2003, A\&A, 406, 969 